\title{
Sindrome de dificultad respiratoria del recién nacido
}

DRA. MAFALDA RIZZARDINI P. *

EI S. D. R. constituye el trastorno más frecuente de observar en el período neonatal inmediato. Se entiende por tal la presencia de taquipnea, retracción y quejido presente desde las primeras horas de vida.

El sindrome de dificultad respiratoria puede ser producido por diversas causas; neumonia connatal, aspiración meconjal, neumotórax, neumomediastino, malformación congénita, etc., pero la más frecuente y la que causa mayor mortalidad en el período neonatal es el Sindrome de Dificultad Respiratoria idiopático o Sindrome de membrana bialina (SRDI o SMH).

Cabe destacar por su frecuencia, aunque no por su gravedad al llamado Sindrome de Dificultad respiratoria Benigno (SDRB) el que es de evolución fugaz y cuyo diagnóstico se hace por exclusión.

En esta oportunidad nos referiremos sólo al SDRI y a algunos aspectos del diagnóstico del SDRB los que en algunas oportunidades son bastante difíciles de distinguir aún con la ayuda del Iaboratorio.

\section{Sintomatologia}

El SDRI es un cuadro clínico que afecta casi exclusivamente a RN prematuros $\left(\begin{array}{lll}1 & \text { y } & 2\end{array}\right)$ y a hijos de madre diabética (3). La sintomatología se inicia inmediatamente después de] nacimiento y va aumentando de intensidad hasta las 48-72 horas para luego regresar en ef curso de una semana o diez días.

Los sintomas más constantes son: quejido expiratorio, retracción esternal y subcostal, polipnea

\footnotetext{
* Departamento de Pediatria, Hospital R. del Rio, Santiago-
}

Rev. Cbilena Pediatría, Vo]. 44, No 6, 1973. (sobre 70/min.), y cianosis que pasa al administrar $\mathrm{O}_{2}$. La auscultación pulmonar revela menor entrada de aire a los pulmones, disminución del mirmullo vesicular y a veces crépitos (4).

Si la enfermedad progresa aumenta el ritmo respiratorio, la retracción y la cianosis. Aparece eđema periférico y disminuye el tono muscular y la temperatura. Pueden aparecer pausas de apnea la que se atribuye a la asfixia cuando son cortas y leves pero que traducen la presencia de hemorragias masivas intracraneales cuando son severas, subintrantes y finales (5).

\section{Laboratorio}

La Rx de tórax en el RN con SDR debe tomarse inmediatamente después de iniciada la sintomatología para descartar otras patologías como: hernia diafragmática, neumotórax, insuficiencia cardíaca. Inicialmente la $\mathrm{Rx}$ puede ser normal pero a medida que progresa la enfermedad aparece el aspecto típico: velamiento difuso simétrico, o no de ambos pulmones con un fino reticulado-granular y broncogramas de aire. Casi constantes también son: el ensanchamiento del mediastino superior y la cardiomegalia (6).

El estudio de gases sanguíneos revela hipoxemia, acidosis respiratoria y acidosis metabólica secundaria a hipoxia.

Un estudio reciente en nuestro medio reveló que los pacientes que mejoran tienen un $\mathrm{pH}$ inicial mayor de 7,20 , en cambio la mayor parte de los niños que fallecen tienen un $\mathrm{pH}$ inicial inferior a 7,20 e incluso bajo 7,10 U. (7).

La $\mathrm{P}_{2} \mathrm{CO}_{2}$ es generalmente inferior a $50 \mathrm{mmHg}$ en los niños que mejoran en cambio los que fallecen tienen retención severa de $\mathrm{CO}_{2}$ la que tiende a aumentar a pesar del tratamiento. 
Las mediciones seriadas de $\mathrm{O}_{2}$ en sangre arterial revela bipoxemia severa con $\mathrm{PaO}_{2}$ en algunos casos inferiores a $30 \mathrm{mmHg}$, aunque estaban respirando una concentración de $\mathrm{O}_{2}$ elevada. Las concentraciones de $O_{2}$ se fueron graduando en este grupo estudiado de acuerdo a las necesidades de cada paciente, sin embargo en los niños que fallecen se observa un deterioro contínuo de la $\mathrm{P}_{0} \mathrm{O}_{2}$ aún respirando concentraciones de 02 cercanas al $100 \%$.

EI componente metabólico de la acidosis aunque más tardíamente que la acidosis respiratoria se observa prácticamente en fodos los niños con SMH. E1 déficit de base varía entre -20 y $-1,5$ $\mathrm{mEq} / \mathrm{L}$ observándose las acidosis metabólicas iniciales más severas entre los niños que fallecen (EB entre -11 y $-20 \mathrm{mEq} / \mathrm{L}$ de sangre).

$\mathrm{El} \mathrm{pH}$ revela la acidosis mixta descompensada la que por supuesto es más intensa e irreversible en los niños que fallecen por S.D.R.I. aunque inicialmente parecería que hubiera cierta tendencia a la normalización, bajo la acción del bicarbonato de sodio.

Las reservas de glucosa se agotan debido al intenso trabajo de los músculos respiratorios y como el prematuro tiene poca grasa, la reoglucogénesis se hace a expensas de las proteínas con la consiguiente lisis celular y liberación de $\mathrm{K}$ en el torrente sanguíneo. Cuando la kalemia sube de $7 \mathrm{mEq} / \mathrm{L}$ hay alteraciones en el ECG las que ceden cuando se suministra glucosa y bicarbonato. (8). Probablemente a este mayor catabolismo se debe también el aumento del $\mathrm{N}$ no proteico y del P. En la orina se observa aumento de los 17 hidroxicorticoides (9-10).

E1 S.D.R.I. frecuentemente se asocia a hipocalcemia debido a que la anoxia estimula la producción de "calcitonina" que aumenta la excreción de Ca por el riñón. La hipocalcemia puede ser también secundaria a la administración de bicarbonato el que disminuye la absorción intestinal y aumenta la eliminación renal (11-12).

\section{Anatomia patológica}

En autopsia de niños que fallecen por MH se encuentran zonas atelectásicas, membranas hialinas, hemorragias intersticiales y/o alveolar y edema cuyo origen puede ser la vasoconstricción arteriolar que se inicia en el feto y período de RN. (5 y 13). La hipoperfusión pulmonar daña el epjtelio alveolar y las células osmiofílicas que producen el "surfactante" que recubre el cpitelio alveolar. La falta de sustancias tenso-activas perturba la expansión pulmonar inicial y la estabilidad de los espacios aéreos después del nacimiento; Bozic (14) encuentra atelectasia y depósitos de fibrina en 4 đe 5 lóbulos de un niño con SDRI, el
Ióbulo de apariencia normal era irrigado por una arteria anómala que nacía de la aorta lo que demuestra que la asfixia local del pulmón fetal juega un papel importante en la génesis del SDRI.

Frecuentemente se encuentra permeable el ductus y el agujero de Botal con un gran shunt de derecha a izquierda lo que se ha atribuído a la presencia de niveles sanguíneos elevados de catecolaminas que relajan el ductus y contraen la arteria pulmonar (15-16).

La hemorragia cerebral es un hallazgo frecuente en los niños por SDR las que se ubican la mayor parte de las veces en los espacios subaracnoideos o ventriculares. Ocasionalmente se encuentran focos de neumonia en los niños que fallecen más tardiamente (5).

\section{Diagnóstico}

El diagnóstico clínico se basa en el análisis cuidadoso de los antecedentes materno-fetales, en el cuadro clínico y en la progresión de la sintomatología que es más importante que su presencia en un momento dado. El cuadro característico del inmaduro, se inicia en el momento del nacimiento o poco después; progresa hasta la $36-48$ hrs., y luego regresa lentamente.

El diagnóstico diferencial se plantea con otros tipos de insuficiencias respiratorias como:

1. Afecciones del SNC que produce apnea o hipopnea, más que dificultad respiratoria. Hay generalmente signos de depresión del centro respiratorio por falta de $\mathrm{O}_{2}$, trauma o hemorragia intracraneana, narcosis, shock post hemorragia, sepsis o anonialías del SNC.

2. Afecciones de las vías aéreas o pulmonares y anomalias torácicas: Cabe destacar el netumotórax a tensión que se manifiesta por disnea, cianosis y falla circulatoria y cuyo diagnóstico se confirma mediante el estudio radiológico. El enfisema lobar congénito, generalmente presente al nacer que produce disnea y cianosis observándose el hemitórax afectado hiperexpandido e inmóvil.

En la hernia diafragmática, generalmente izquicrda, la sintomatología aparece inmediatamente después del nacimiento observándose disnea, cianosis y a veces vómitos. La auscultación pulmonar puede revelar la presencia de las vísceras abdominales que han pasado al tórax por la brecha diafragmática y la desviación del corazón hacia el lado derecho.

La atresia de los coanas es una afección rara que produce dificultad respiratoria grave cuando es bilateral. Los síntomas pasan al abric la boca, y el diagnóstico se hace pasando una sonda nasal.

La bronconeumonia connatal inicialmente no da sintomatología muy específica pero a las pocas horas de vida, al SDR progresivo se agrega pér- 
dida de vitalidad, palidez terrosa y síntomas gastrointestinales. La auscultación pulmonar revela signos de condensación, especialnente crépitos.

La aspiración amnio meconial produce distress desde el nacimiento, existe siempre el antecedente de sufrimiento fetal con pasaje de meconio al hiquido amniótico y cs raro de observar en cl niño inmaduro. El SDR es extremo porque el meconio produce obstrucción e inflamación del pulmón. La función respiratoria se normaliza, en los sobrevivientes, al 3-4 días aunquc puede persistir polipnea durante 8 a 10 días y alteraciones radiológicas mínimas.

El estudio radiológico demuestra la existencia de áreas de opacidad "floculentas", zonas con superaereación, neumotórax y a veces neumomediastino.

3. Sindrome de hiperviscosidad sanguínea. La policitemia con hematocrito superior a $70 \%$ puede asociarse a taquipnea y distress respiratorio. Lil Rx. revela cardiomegalia y congestión vascular pulmonar. Generalmente se trata de niños con suftimiento fetal crónico que evidencian al nacer, signos inequívocos de desnutrición intrauterina, afecta de preferencia a los varones y se asocia a hipoglicemia, a síntomas del SNC como apatía, temblores, convulsiones y a priapismo (17-18).

Como vemos el diagnóstico clínico del SDRI es bastante dif́cil cuando no se toman en cuenta la historia obstétrica, el examen físico del paciente y la evolución de la sintomatología. El diagnóstico exacto sólo puede hacerse mediante la ayuda del radiodiagnóstico y aún así quedan dudas que sólo pueden disiparse si el niño fallece.

Según Usher (19) en los casos típicos de $\mathrm{MH}$ la $R x$ no es necesaria ya que él encuentra $100 \%$ de correlación clínico radiológica. En los niños a término y cuando no se ha observado al prematuro desde las primeras horas de vida la radiografía es de gran utilidad y sirve más que para identificar alteraciones "típicas" para eliminar otros diagnósticos. (6).

Más difícil aún cs separar el SDRI del llamado Distress Respiratorio Benigno (DRB) o jnicial ya que si el SMH es muy leve puede ser clínicamente indistinguible. La cuantificación de sustancias tenso-activas cn la secreción braqueal es un avance promisor en el diagnóstico del SDRL (22).

El DRB se relaciona con edema intersticial del pulnón pero es posible que muchos casos sean SRDI frustros; Avery llama a este cuadro "Taquipnea transitoria del RN" (TT) y reporta 8 casos en RN a término con taquipnea, retracción moderada y quejido en el primer día de vida, que persiste 2 a 5 días. La $R x$ da hiperaireación y aumento de la trama broncovascular (23). Kuhn y Swischuk, cncuentran líquido plcural en $\mathbf{1 8}$ casos similares en los que se observan pequeños cambios en el equi- librio ácido básico y gases sanguíneos, los que desaparecen en 12-24 horas (24-25).

Prod'honi (26) crec que el DRB o inicial como también lo llama, a diferencia del SDRI tiene escaso shunt de derecha a izquierda, aunque en ambos grupos hay menor índice de ventilación/perfusión, menor ventilación alveolar y menor elasticidad pulmonar. Las alteraciones son transitorias en el DRS y mejoran pasadas las 24 horas. Podría especularse que tanto la TT del RN como el DRB y el SDRI representan grados de anormalidad en la adaptación del pulmón al intercambio y probablemente los mecanismos patogénicos sean los mismos pero los resultados diferentes, según sean los factores contribuyentes y sobre todo, según sea el grado de madurez pulmonar del recién nacido.

\section{Etiología}

Hay numerosas tesis para explicar el SDRI. Algunos autores han encontrado cierta relación entre cl nivel de enzimas fibrinolíticas en el pulmón y en la sangre y mebranas hialinas pulmonares. (27). Otros han encontrado un inhibidor del activador del plasminógeno el que sería el responsable del trastorno pero hay estudios que contradicen estos hallazgos por lo que se les ha restado importancia (28).

Aparentemente lo más importante es la alteración o disminución del surfactante pulmonar que es el encargado de mantener la estabilidad alveolar después de la primera inspiración. Lo que aún se discute es si este déficit es primario y en relación al grado do madurez del niño o es secundatio a la isquemia pulmonar que resulta de la asfixia intrauterina.

El surfactante es un complejo formado por proteínas, hidratos de carbono, pero especialmente lípidos siendo el principal componente la lecitina, de alto poder para bajar la tensión superficial (29).

El surfactante impide que los alvéolos se colapsen totalmente en la expiración por lo que el déficit de esta sustancia produce atelectasia. El colapso pulmonar condiciona hipoxia, acidosis, vasoconstricción pulmonar, menor producción de surfactante y mayor colapso. La hipoxia daña el endotelio capilar y se produce transudación de líquido con fibrina hacia la luz del alvéolo que es lo que origina las membranas hialinas.

Se describen dos pasos enzimáticos en la biosíntesis del surfactante.

Fase I. Sintetiza dipalmitol-lecitina baja la acciốn de la fosfocolinotransferasa. En el ser humano esta etapa madura a las 35 semanas de gestación y en otros mamíferos sólo después que el feto comienza a respirar (30).

La fase II origina un producto activo, el palmitol-miristol lecitina bajo la acción de una metilttransferasa (31). La reacción se identifica desde 
las 22-24 semanas la gestación y se completa al término, constituyendo al final, la mitad del surfactante. Esta sustancia permite la sobrevida del prematuro después de 24 semanas siempre que al nacer sintetice rápidamente mayor cantidad de sustancias tensoactivas (30). En las ratas, conejos y corderos cuyo surfactante madura sólo en los últimos días de gestación no se encuentra este tipo de fosfolipidos.

El SRDI sería por lo tanto la consecuencia directa de la inmadurez pulmonar desencadenado por factores que interfieren en la sintesis rápida del surfactante en el momento del nacimiento. Aparentemente Ia acidosis no sólo interfiere en la producción de las sustancias tensoactivas al inducir vasoconstricción pulmonar sino que actúa directamente inhibiendo la fase de metilación del proceso enzimático que termina en la sîntesis del surfactante, cerrando así el círculo vicioso al producir atelectasia, mayor hipoxia, etc. (31).

\section{Prevención}

La prevención del SDRI es la de la prematumridad ya que es un trastorno que afecta casi exclusivamente a $\operatorname{los} \mathrm{RN}$ inmaduros. Cuando ello no es posible es necesario evitar los accidentes del parto que frecuentemente se asocian a MH (asfixia, hemorragias, anestesia y analgesia inadecuadas); tratar la hipoxemia, acidemia e hipotensión maternas para prevención la asfixia neonatal; evitar enfriamientos en la sala de partos para prevenir acidosis metabólica y vasoconstricción pulmonar; inyectar $\mathrm{Na} \mathrm{HCO}_{3}$ al RN asfixiado para aumentar la contractibilidad cardíaca, la vasodilatación pulmenar y reducir el daño cerebrat. Mantener al nir̆o en un ambiente térmico neutral para disminuir los requerimientos de $\mathrm{O}_{2}$ al mínimo.

Una vez establecida la enfermedad sólo se pueden corregir las alteraciones de la homeostasis, corrección que debe basarse en el conocimiento exacto de la fisiopatología del proceso.

Ultimamente se ha ensayado el uso de corticoides para acelarar la maduración del pulmón fetal en aquellos casos en los que el parto prematuro es inevitable.

Liggins (32) en 382 madres cuyo parto se planeaba para antes de las 37 semanas de gestación administra betametazona $6 \mathrm{mg}$. diarios observando un significativo descenso de la mortalidad perinatal. El SDRI fue mucho menos frecuente en el grupo tratado que en el control ( $19 \%$ contra $25,8 \%$ ).

El corticoesteroide administrado al njĩo después del nacimiento no previene ni modifica el SDRI (33-34) aunque Altman (35) en un grupo pequeño señala que el corticoesteroide también sería beneficioso cuando se administra al niño después del nacimiento. Podría intentarse su uso en RN de 26 a 32 semanas en los que la morbilidad y mortalidad por SRDI son realmente alarmantes.

Los corticoides aceleran la maduración pulmonar del feto tanto en corderos como en conejos (36-37-38) de experimentacion. Pocos corderos prematuros tienen atelectasia pulmonar $y$ las sustancias osmiofílicas aumentan o aparecen en conejos prematuros cuando se ha administrado corticoides a la madre antes del parto, por lo que se cree que el corticoesteroide aunque no sea el mediador enzimático natural del surfactante induce la producción de la enzima fosforil colina-glucoride transferasa que aumenta la síntesis de la lecitina (38-39).

Las suprarrenales del feto son capaces de producir cortisona como reacción normal ante cualquier stress pero la concentración de 17 hidroxicorticoesteroides en el cordón es el reflejo del nivel materno el que a su vez depende del grado de stress al nacimiento. El cortisol atraviesa la placenta manteniendo concentraciones plasmáticas en el feto cercanas a $10 \mu \mathrm{g} / 100 \mathrm{ml}$. Durante el parto vaginal estos niveles suben a $30+\mathrm{g} / 100 \mathrm{ml} \pm \mathrm{DS}$ $5 \mathrm{mg}$. cosa que no ocurre en el parto por cesárea no precedido de trabajo (40).

El convencimiento de que el riesgo de $\mathrm{MH}$ disminuye en los prematuros cuya cesárea va precedida de trabajo de parto hace suponer, a la luz de estos nuevos conocimientos, que existe cierta relación entre trabajo de parto, corticoesteroides y maduración pulmonar. También se ba visto que (41) Jas suprarrenales de los niños con $\mathrm{MH}$ son $19 \%$ más livianas que las de los niños sin problemas lo que podría hacer suponer que producen menos corticoesteroides y no lo suficiente para inducir maduración pulmonar adecuada. Sin embargo Reynolds (42) ba demostrado que los niveles séricos de corticoides y cortisol de los recién nacidos con SDRI fatal son significativamente más altos que los de los niños con SDRI no fatal o que padecen de SRDB lo que indicaría que si hay hipofunción suprarrenal en el niño con SDRI, ésta no es postnatal. Los anencefálicos que tienen suprarrenales muy pequeñas tienen la mitad de los gránulos osmofílicos en el pulmón comparado con niños normales de la misma edad gestacional. Por otro lado las suprarrenales de los niños con infección antenatal tienen la zona adrenal que produce corticoides tan grande como la de los adultos probablemente como respuesta al stress de la infección (41). Estos niños rara vez sufren de membrana hialina cualquiera que sea su edad gestacional, lo que podría significar que las infecciones bacterianas estimulan la producción de corticoesteroide y ésta la maduración pulmonar.

\section{Tratamiento}

E] tratamiento de Usher ha demostrado ser efectivo para reducir la mortalidad por MH (43). 
La glucosa debe dar suficientes calorías para evitar el catabolismo exagerado, la hiperkalemia y la acumulación de cuerpos cetónicos.

La acidosis mixta preferentemente metabólica, es un potente vasoconstrictor pulmonar lo que disminuye la sintesis del surfactante, aumenta el shunt de derecha a izquierda y afecta por lo tanto la ventilación/perfusión y también la difusión. Se ha demostrado además que la acidosis disocia la bilirrubina de la albúmina y aumenta la afinidad de las mitocondrias cerebrales por el pigmento, lo que predispone al kernicterus (44).

Por estas razones el $\mathrm{NaHCO}_{3}$ que además de corregir la acidosis metabólica es un vasodilatador arteriolar, debe administrarse precozmente en cantidades que varían entre 5 y $15 \mathrm{mEq} / 100$ al suero glucosado al $10 \%$ según sea la magnitud de la acidosis.

El THAM que tiene el mismo efecto y produce un cambio más rápido a la alcalosis, sólo se recomienda cuando la cantidad total de bicarbonato a administrar es tan grande que existe riesgo de producir hipernatremia ya que este alcalinizante produce hipoglicemia, crisis de apnea, disturbios electrolíticos e irritación tisular (45). El lactato no se metaboliza en el prematuro. El bicarbonato por vía oral es de absorción muy incierta $y$ no produce mucho efecto.

\section{Termorregulación}

De igual importancia es la mantención de la temperatura corporal del niño ya que el frío induce mayor entrega de norepinefrina la que produce vasoconstricción pulmonar, disminución de la $\mathbf{P}_{4} \mathrm{O}_{2}$ y aumento de los ácidos grasos no esterificados que compiten con los aniones como la bilirrubina en su unión con la albúmina.

Para el consumo de $\mathrm{O}_{2}$ sea mínimo hay que mantener en un ambiente térmico neutral que en el RN es de $32^{\circ}$ a $34^{\circ}$. En el RN normal la temperatura ambiental baja aumenta la ventilación menuto pero en el DR el ritmo respiratorio está sobre 100 por minuto y no se puede aumentar mucho más por lo que hay tendencia al enfriamiento (47-48-49).

Las pérdidas de calorías por evaporación pulmonar y cutánea se obvian con el ambiente húmedo de la incubadora y evitando la aplicación de $\mathrm{O}_{2}$ frío no humidificado sobre la cara o en máscara $(50)$.

\section{Oxigenoterapia}

Para la administración de $\mathrm{O}_{2}$ en el SDR bay que guiarse por la cianosis. El color azul de la piel rara vez aparece en el $\mathrm{RN}$ antes que la $\mathrm{P}_{\mathrm{k}} \mathrm{O}_{2}$ caiga de $60 \mathrm{mmHg}$ y a veces de 50 debido a la posición de la curva de disociación de la $\mathbf{I H b}$ en el RN. Pero no quiere decir que no haya hipoxenmia tisular aún con buena coloración. El mínimo de tensión de $0_{2}$ capilar necesario para una adecuada oxigenación tisular es, en prematuros, de $30 \mathrm{mmHg}$, variando el múnimo de acuerdo a los requerimientos propios de cada tejido.

En ocasiones no es suficiente el uso de concentraciones de $\mathrm{O}_{z}$ del $40 \%$ que es lo que da la incubadora, entonces hay que llegar a concentraciones de $100 \%$ del aire inspirado.

$\mathrm{El} \mathrm{O}_{2}$ administrado en altas concentraciones ha demostrado ser tóxico para algunos tejidos como la retina y el pulmón. En el SDR el peligro de dañar la retina es sin embargo mínimo ya que el daño no depende de la concentración de $\mathrm{O}_{2}$ del aire inspirado, sino de la $\mathrm{P}_{2} \mathrm{O}_{2}$. En la neumonia en que sólo hay un defecto en la difusión la administración de altas concentraciones de $\mathrm{O}_{z}$ puede fácilmente determinar $\mathrm{P}_{0_{2}} \mathrm{O}_{2}$ en Ia sangre muy elevadas, pero en el SRD donde además de un defecto de difusión hay también un trastorno de la perfusión con un gran shunt de derecha e izquierda difícilmente la $\mathrm{P}_{2} \mathrm{O}_{2}$ puede alcanzar límites peligrosos, capaces de dañar las arterias retinianas, en las que produce vasoconstricción.

Recientes estudios, sin embargo, han demostrado que existe una gran variabilidad en la $\mathrm{P}_{\mathrm{a}} \mathrm{O}_{2}$ capaz de producir espasmo arteriolar en la retina $100 \mathrm{~mm} \mathrm{Hg}$ o menos pueden originar fibroplasia retrolental y $400 \mathrm{~mm}$ no tener efecto alguno deletereo. Probablemente entren en juego otros factores como: grado de inmadurez, duración de la administración de Os, hipoxia previa, cambios bruscos de $\mathrm{P}_{6} \mathrm{O}_{2}$, etc. (51-52).

En el pulmón Ja hiperoxigenación produce cambios anatómicos de los capilares pulmonares con microhemorragias difusas, disminución en la producción de mucus e inactivación del surfactante. (51-52). Recientemente se ha observado un efecto inhibidor del $\mathrm{O}$ sobre la síntesis del DNA del pulmón (55). En este órgano el $\mathrm{O}_{2}$ actúa en forma directa independiente de la $\mathrm{PAO}_{\mathrm{A}}$ produciendo una displasia broncopulmonar que sólo se observa cuando se usan respiradores a presión positiva e intubación endotraqueal, pero rara vez cuando se usan respiradores a presión negativa sin tubos endotraqueales aunque Ia concentración de $\mathrm{O}_{2}$ sea muy alta y por largos períodos (56-57-58-59).

La oxigenoterapia extracorpórea podría ser un procedimiento útil y se ha usado en forma experimental pero no se conocen los resultados.

En todo caso parece evidente que la oxigenoterapia aunque es un procedimiento salvador puede en algunos casos, ser un arma de doble filo cuyo uso requiere un adecuado monitoreo del paciente. Nuevos procedimientos se han ideado para evitar o pesquisar precozmente la hiperoxemia, 
desde el simple test en sangre de la arteria umbilical hasta la colocación de clectrodos en el tóbulo de la oveja o la medición de la tensión de $\mathrm{O}_{2}$ de la conjuntiva palpebral pero no es posible dotar a todos los centros de estos métodos sofisticados de control. Más recomendable es usar la oxigenoterapia como criterio para el traslado de un niño de cualquier sitio a un centro de cuidado intensivo cn el que se disponga de todos los elementos necesarios.

\section{Otras terapéuticas}

Numerosos procedimientos se han usado para mejorar el pronóstico de la membrana hialina. El dipalmitol lecitina se ha usado en aerosol pero no ha tenido efecto probablemente porque no llega el alvéolo colapsado o porque se inactiva (60). Tampoco sirven los fibrinolíticos en aerosol o endovenoso porque no remueven las membranas hialinas formadas (27).

La acetil colina para tener efecto, tendría que administrarse directamente en la circulación pulmonar para no ser inactivada por la colinoestearasa al ser izyectada por una vena periférica. Es un medicamento peligroso porque produce hipotensión grave y súbita (61).

El recambio sanguíneo con sangre produce mejorias espectaculares aunque transitorias de la oxigenación del RN con SDRI especialmente en los niños con anemia asociada, pero el procedimiento aunque tiene bases teóricas es peligroso en niños tan enfermos.

\section{Pronóstico}

Con el mejor de los tratamientos actuales el $50 \%$ de los pacientes fallece, 4 a 5 lo hacen entre las 12 y 72 horas. De los que sobreviven más de 48 hrs., fallece el $25 \%$; de los que sobreviven más de 72 hrs., el $11 \%$ (19).

A medida que aumentan los esfuerzos para lotar a los centros regionales de los últimos odelantos para el manejo del SDRI, especiámente de respiradores a presión positiva, sc hace más nece. sario el identificar lo más pronto posible al nin̄o de alto riesgo mediante datos objetivos y precisos.

Se sabe que la letalidad es más elevada en el virón que cr la mujer, en el gemelo segundo que en el primero y que existe una relación directa entre madurez fetal y gravedad del SDRI (2).

La madurez pulmonar del feto puede estimarse identificando en el líquido amniótico dos sustancias con propiedades tensoactivas: lecitina y esfingomielina (33-62-63). La relación entre estos dos fosfolípidos es un buen indicador de maduración pulmonar fctal. La concentración de lecitina en ell líquido amniótico en las primeras sc- manas de gestación es menor que la de esfingomiclinil. Entre las 30-32 semalnas ambos compucstos se encueotran en igual cantidad. A partir de las 35 semanas comienza a aumentar la lecitina la que, al final de la gestación excede con mucho a la esfingomielina (64).

La medición del índice lecitina-esfingomielina constituye por lo tanto un buen elemento pronóstico no sólo para predecir la aparición del SDRI, sino que también para pronosticar su gravedad, la que a su vez depende del grado de madurez pulmonar del recién nacido (63-65).

Hay algunos trastornos maternos y placentarios que aceleran la maduración del surfactante y modifican por lo tanto la relación entre lecitina y esfingonielina: toxemia severa, enfermedades renales, cardiovasculares, sangramientos placentarios, ruptura prematura de membrana, infecciones congénitas, uso de esteroides al final de la gestación, de hormonas tiroides y adición de heróna. $(65,66,67,68,69,70)$.

Por otro lado la diabetes materna y la enfermedad hemolítica grave retardan la maduración pulmonar, observándose aumento rápido de la lecitina sólo al final de las 37 semanas de gestación. (65).

Desgraciadamente la amiocentesis es una técnica que sólo puede aplicarse a un número muy reducido do embarazadas por lo que se siguen buscando otros métodos para poder identificar precozmente a este grupo de recién nacidos de alto riesgo.

Brand ha cuantificado las proteinas totales del cordón en 2.200 recién nacidos observando que un tcrcio de todos los niños y la mitad de los prematuros que tienen proteínas bajo $4,7 \mathrm{~g} / 100 \mathrm{ml}$. en la sangre del cordón, padecen de SDRI en el período neonatal. La incidencia de SDRI en los niños con niveles sanguíneos de proteínas sobre $4,6 \mathrm{~g} / 100$ es sólo de $0,5 \%$ independientemente del grado de madurez. Podría ser este examen un buen y fácilmente reproducible indicador de riesgo de enfermar y/o morir por SDRI (71).

Después del nacimiento la composición de los fosfolípidos pulmonares pueden estimarse en la secreción traqueal mediante cromatografía. La presencia en cl mucus de la vía respiratoria de sustancias con alto poder tensoactivo es un buen indicador de maduración pulmonar. Tanto en la experiencia de Gluck como en la de Wu, la dosificación de estas sustancias es de gran utilidad en el diagnóstico y pronóstico del SDRI. (22-73).

Se ha demostrado que una vez establecido ef sindrome hay algunos exámenes de laboratorio que tienen cierto valor pronóstico. Los niveles inicioles de $\mathrm{PaO}_{2}$ bajo $50 \mathrm{mmHg}, \mathrm{pH}$ inferior a $7,20 \mathrm{U}$. $\mathrm{P}_{4} \mathrm{CO}_{2}$ sobrc $60 \mathrm{mmHg}$ y déficit de base mayor de $10 \mathrm{mEq} / L$ son de muy mal pronóstico sobre todo si no se observa tendencia rápida a la nor- 
malización de estos índices bajo la acción del tratamiento. La presencia de hipoxemia y acidosis bajo niveles críticos, aún después de la administración de elevadas concentraciones de $\mathrm{O}_{2}$ demuestra la presencia de un shunt de derecha a izquierda de considerable magnitud a nivel pulmonar y cardíaca y la gran retención de $\mathrm{CO}_{2}$ indica que existe un grave trastorno de la relación ventilación-perfusión y también de la difusión. La mantención de estos trastornos lleva a alteraciones electrolíticas, hormonales y hemodinámicas de muy difícil o imposible manejo y son por to tanto, de muy mal ponóstico.

Existen pocos estudios a largo plazo en los niños que han padecido de SDRI. Stahlman (74) en 85 casos seguidos durante seis años no observa diferencias en el desarrollo emocional, QI y defectos, comparados con un grupo control. Sólo en un niño encuentran cambios mínimos en la radiografía pulmonar sugestivos de fibrosis. Siete tienen leve déficit de la función pulmonar (dos de tipo obstructivo y cinco restrictivo). Uno requirió tratamiento quirúrgico por un ductus arterioso persistente. Ninguno tiene fibroplasia retrolental a pesar que 21 de ellos padecían de un sindrome de membrana hialina tan grave que requirió ventilación asistida con respiradores de presión negativa $y$ ocasionalmente positiva.

\section{REFERENCLAS}

1.-Dunn, $P$. The respitatory distress syndrome of the newborn: Immaturity vs, prematurity. Arch. Dis. Child. 40 : $62,1965$.

2.-Rizzardini, M., Cerun, E. Sindrome de dificultad respiratoria idiopática. I. Aspectos clînicos. Pediatría (Sontiago) 13: 27, 1970.

3.-Cellis, $S$, and Hsia. D. The infant of diabetic mother.. Aner. J. Dis. Child. 97: 1, 1959.

4.--Rudolph, A. Desmond, $M$. and Pineda, R. Clinical diagnosis of respiratory difficulty in the newborn. Pediat. Clin. M. A. 13: 669, 1966.

5.- Lauweryns, J. Hyaline membrane disease. A pathological study of 55 infants Arch. Dis. Child. 40: 618,1965

6.-Weller, $M$. The roentgenographic course and complications of hyaline membrane disease. Pediat. Clin. N. A. 20: 38I, 1973.

7.-Cerui, E, y Rizzardini, M. Sindrome de dificultad respiratoria idiopática II. Aspectos metabolicos. Rev, Crile. Pediat. 42: 519, 1971.
8.- Usiner, $R$. The respiratory distress syndrome of prematurity 1. changes in potassium in the serum and the electrocardiograma and effect of therapy. Pediatrics 24: 562, 1959.

9.-Usher, $R$. The metabolic changes in respiratory distress syndrome of prematurity seen as a failure of somatic compensations for asphyxia. Ciba Symposium on somatic stability in the newborn. London-Cburchill, 1961.

10.- Lim, $N$. Algunos aspectos de la endocrinologia del feto y il neonato Clin, Pediat. N. A. Noyicmbre, 1966.

11.-Copp, D. Endocrine, control of calciun homeostasis, J. Endocrinol, 43: 137, 1969.

12.-Stem, $L$. Therapy of the respiratory distress syndrome Jediat. Clin, N. A. 19: 221, 1972.

13.-Driscoll, S. and Smith, C. Neonatal pulmonary disorders. Pediat. Clin. N. A. 9: 325, 1962.

14.-Bozic, C. Pulmonary hynline membranas and vascular anomalies of the hung. Pediatrics 32: 1092, 1963.

11.- Copp, D. Endoctine, control of calcium homeostasis, J. Endocrinol. 43: 137, 1969.

12.-Stern, L. Therapy of the respiratory distress syndtome Pediat. Clin. $N^{*}$ A. 19: 221, 1972.

13.-Driscoll, S. and Smith, C. Neonatal pulmonary disorders. Pediat. Clin. N. A. 9: 325, 1962.

14.-Bozic, C. Pulmonary hyaline membranes and vascular anomalies of the hung. Pediatrics 32: 1092 , 1963.

15.-Chu, J. Cloments, J. Cotton, E. Neonatal pulmonary ischemia. Pediatrics 40: 709, 1967.

16.-Rudolph, A. Drorbaugh, J. Audl, P. Studies on the circulation in the neonatal period. The circulation in the respiratory distress syndrome. Pediatrics 27: 551, 1961.

17.-Bravo, $M$. Valenzuela, $J$, y Rizuardini $M$. Sindrome de hiperviscosidad sanguínea en el período neonatal (SHS) Pediatría 16: 7, 1973.

18.- Humbert, J., H. Hathaway, W. and Battaglia, F. Polycylhemia in small gestacional age infants. $J$. Pediat. 75: 812, 1969.

19.-Usher, $R$. Clinical investigation of the respiratory distress syndrome of premature. N. State J. Med. 61: $1677,1961$. 
20.- Sundell, H. Garrot. J. Klankenship, W. Shepard, F. and Stakiman, $T$. Studies on infants with type II Jespiratory distress syndrome.

21.-Taylor, P. Allern, A, and Stinson D. Benign unexplained respiratory distress of the newborns infant. Pediat. Clin. N. A. 18: 957, 1971.

22.-Wu, P. Borer, $R$. and Modalou, H. Diagnosis of the respiratory distress syndrome by the absence of phosphatidy e dimethylethanolamine in tracheal effuents of low birth weight infants. Pediat. Res. 5: $415,1971$.

23.-Avery, M. Gatewood, O. and Brumley, G. Transient tachypnea of the newborn. Amer. J. Dis. Child. 111 ; 380,1966 .

24.-Kuhn J., Fletcher, B, and De Lemos, R. Roentgen findings in transient tachypinea of the newborn. Radiology 92: 751, 1969.

26.- Prod'hom, L. Levison, H., Cherry, R. and Smixh $C$. Adjustment of ventilation intrapulmonary gas exchange acid base balance during the first day of life. Pediatrics 35: 662, 1965.

27.-Ambrus, C. W'eintraub, L, Dunply, D. Doud, J. Pickren, J. Niswander $K$. and Ambrus, J. Studies on hyaline membrane disease. The fibribolysis system in pathogenesis and therapy. Pediatrics 32: 10, 1963.

28,- - Netson, $N$. On the etiology hyaline membrane disease. Pediat. Clin. N. A. 17: 943, 1970.

29.-Avery, M. and Mcad, J. Surface properties in relation to atelectasis and hyaline membrane disease. Amer. J. Dis. Child. 95: 517, 1959.

30.-Gluck, L. Kulovich, M. Eidelman, A. Biochemical activity in mamalian lung IV. Pediat. Res. 6: 81, 1972.

31.-Gluck, L. Surfactant. Pediat. Clin. N. A. 19: 325, 1972.

32.-Liggins, G. Controlled trial of antepartum glucocorticoid treatmet for prevention of the respiratory distress syndrome in premature infants. Pediatrics 50: $315,1972$.

33.-Baden, M. Baner C. Colle, E. Klein, G. Taendh, $W$. and Siern, L. A Controlled trial of hydrocortisone therapy in infants with respiratory distress syndrome. Pediatics 50: 562, 1972.

34.-Hadded, H. Hsia, D. and Gellis, S. Studies on respiratory rate in the newborn. Its use in the evaluation of respiratory distress in infants of diabetic mother. Pediatrics 17: 204, 1956.
35.-Aliman, $H$. The respiratory distress syndrome of the newborn. S. Asf. Med. J. 39: 746, 1965.

36.- Ligyins, $G$. Premature delivery of foetal lambs enfised with glucocorlicoid. J. Endocrinol. 45: 515, 1970.

37.- Detemos, R. Shermeta, D. Knelson, J. Kotas R. and Avery, $M$. Acceleration of appearance of pulmonary surfactant in the fetal lamb by administration of glucocorticoids. Amer. Rev. Resp. Dis. 102: $459,1970$.

38.- Kotas, R. and Avery $M$. Accelerated appenrance of pulmonary surfactant in the fetal rabbit. J. Appl. Physiol. 30: 358, 1971.

40.- Stevens, 3. Plasma cortisol levels in the neonatal period. Arch. Dis. Child. 45: 592, 1970.

41.- Naeye, R., Harcke, R. and Blanc, W. Adrenal gland structure and the development of hyaline membrane disease. Pediatrics 47: 650, 1971.

42.- Reymolds, I. Serum total corticoid and cortisol kevels in premature infants with respiratory distress syndcome. Pediatrics 51: 884, 1953.

43.-Usher, R. Reduction of mortality from respiratory distress syndrome of prematurity with early administration of intravenous giucose and sodium bicarbonate. Pediatrics. 32: 966, 1963.

44.- Odell, $G$. The influence of $\mathrm{pH}$ on the distribution of bilirubin between albuminum and mitrochondria. Proceedings of the society for Pediatrie Research Seatlle. Washington, 1964.

45.-Hutchison, J, Kerr, M. Douglas, T, Inall, J. and Crosbie, J. A therapeutic approach in 100 cases of the respitatory distress syndrome of the newborn infant. Pediatrics 33: 956, 1964.

46.-. Schiff, D. Stern, $L$, and Leduc, J. Chemical thermogenesis in newborn infant. catecholomine excretion and the plasma non-esterified fatty acid response 10 cold exposure. Pediatrics 37: 577, 1966.

47.-Bluck, $K$. Temperature regulation in the newborn. Infant. Biol, Neonant. 3; 65, 1961.

48.- Ceruti, E. Torres, J. Díaz, A., Auti G. Bailey, P. y Núriez $N$. Efecto de la temperatura ambiental en la regulación del equilibrio ácido básico en recién nacidos de término y prematuros, en los primeros días de vida. Bol. Med. Hospital Infant. 29: 757, 1972. 
49.- Privylova, $M$. The effect om body temperature on the level of carbohydrate metabolites and oxigen consumption in the newborn. Pediatrics 37: 743, 1966.

50.-Mestyar, J. Jarat, L. Bata, G. The significance of facial skin temperature in the chemical heat regulation of premature infants. Biol. Neonat. 7: 243, 1964.

SI.-A Aanda, J. Saheb, N. Stern, L. Arterial oxygen tension and retinal vasoconstriction in newborn infant. Anter. J. Dis. Child. 122: 189, 1971.

52.-Kalita, R. Hodson, W. and Morgan, B. Retrolental tibroplasia in a cyanotic infant. Pediatrics. 50: $765,1972$.

53.- Senior, R. Hodson, Wesseler, S. and Avioli, L. Pulmonary oxygen therapy toxicity J. A. M. A. 217: 373, 1971.

54.-Giammona, S. Kerner, D. and Boudurant, S. Effect of pulmonary oxygen breathing at atmospheric pressure on pulmonary surfactant. J. Appl. Phy. siol. 20: 855,1965 .

55.-Northway, W. Petriceks, R. and Shahinian, L. Quantitative aspects of oxygen toxicity in the newborn. Inhibition of lung DNA synthesis in the mouse. Pediatrics 50: 67, 1972.

56.- Northway, W. Rasath, R., Porter, D. Pulmonary disease following respirator therapy of hyaline membrane disease. N. Engl, J. Med. 276: 357, 1967.

57.-Ballard, R., Kraybill, E. Hernúndez, J. Renfield, $M$. and Blankenship, $W$. Idiopatibe respiratory distress syndrome. Treatment with continuous negntive pressure ventilation. Am, J. dis. Child, 125: $676,1973$.

58.- Shepard, F. Residual pulmonary finding in clinical hyaline nembrane diseadse N. Engl. J. Med. 279: 1063, 1968.

59.-Chermich, V. and Vidyasagar, D. Continuous negative chest wall pressure in hyaline membrane disense. Pediatrics 49: 753, 1973.

60.-Robilland E. Alarie, Y, Dagenais - Parrusse, P. Baril, E. and Guilbeanlt, A. Micro-aerosol administration of synthetic dipalmetoly lecithin in the respiratory distress syndrome. Canadian Med. Assoc. J. $90: 55,1964$.
61.- Eshoghpour, E. Matliold, L. Williams, M. and Mosilud, A. Acetyloholine in the trealment of idiopathic respiratory distress syndrome. J. Pediat. 71: $243,1967$.

62.- Kotas, $R$. Estimation of pulmonary naturity. J. Pediat. 81: 378, 1972.

63.-Ghack, L. Kulowich, M. Bore, R. Brenner P. A Hr derson, $G$. and Spelfacy, $W$. Diagnosis of the respiratory distress syndrome by ammiocentesis Amer. J. Obstet. 109: 440, 1971.

64.-Gluck, L. Kulowich, M., Borerr, R. Brenner, P., Ardenson $G$. and Spellacy $W$. Diagnosis of the respiratory distress syrudrome by ammiocentesis. Amer. J. Obstet. Gynec. 109: 440, 1971.

65.-Gluck, L. and Kulowich, M. Lecithin / sphingomyelin raties in amniotec in normal and abnormal pregnancy. Amer. J. Obstet. Gynec. 115: 539, 1973.

66.-Gluck, L., Kulovich, M. and Gould J. The effects of maternal disease on naturation of buman fetal lung. Proceedings of the society for Pediatrics Research 42nd Annual Meeting. Washington, 1972.

67.-Motoyamma, E. KiKKawa, Y. Orzalesi. M. Kaibara M. Wa B. Zigas, C. and Cook, D. Efect of corticol on maturation of fetal rabbit lungs. Pediatrics 48: 547,1971 .

68.-Taensk, H. Heiner, M. and Avery, M. Accelerated lung maturation and increased survival in premature rabbits tranted with hydrocortisame Amer. Rev. Resp. Dis. 105: 971, 1972.

69.-Reddung, R., Douglas, $W$. and Stein, $M$. Thyroid hormone influence upon lung surfictant metabolism. Science 175: 994, 1972.

70.- Glass, L., Rajegowda, B. and Evans, H. Absence of RSD in premature infants of heroin nddicted mothers. Lancet 2: 685, 1971.

71. - Brand, $R$, Cord, Blood. Total protein level as a screening aid for the idiopathic respiratory- distress syndrome. New Engl, J. Med. 287: 9, 1972.

72,-Gluck, L. Kulovich, M. Eidelman, A. Biochemical development of surface activity in mammalinn lung. Pediat. Resp. 6: 81, 1972.

73,-Stahlman, M. Hedwall, G. Delanski, El Faxelius, G. Burks $H$. and Kirk, W. A six year follow up of clinical hyaline membrane disease Pediat. Clin. N. A. 20: 433, 1973, 